\title{
A Case Study of On-the-fly Wide-field Radio Imaging Applied to the Gravitational Wave Event GW151226
}

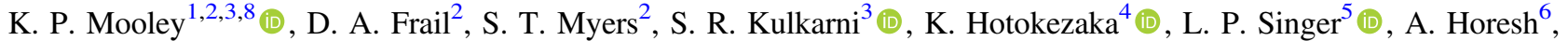 \\ M. M. Kasliwal ${ }^{3}$ (D) S. B. Cenko ${ }^{5,7}$ (D), and G. Hallinan ${ }^{3}$ \\ ${ }^{1}$ Denys Wilkinson Building, Keble Road, Oxford OX1 3RH, UK; kmooley@caltech.edu \\ ${ }_{2}^{2}$ NRAO, P.O. Box O, Socorro, NM 87801, USA \\ ${ }^{3}$ Caltech, 1200 E. California Blvd., MC 249-17, Pasadena, CA 91125, USA \\ ${ }^{4}$ Department Astrophysical Sciences, Princeton University, Peyton Hall, Princeton, NJ 08544, USA \\ ${ }^{5}$ Astrophysics Science Division, NASA Goddard Space Flight Center, Mail Code 661, Greenbelt, MD 20771, USA \\ ${ }^{6}$ Racah Institute of Physics, Hebrew University of Jerusalem, Israel \\ ${ }^{7}$ Joint Space-Science Institute, University of Maryland, College Park, MD 20742, USA \\ Received 2018 January 4; revised 2018 February 27; accepted 2018 March 15; published 2018 April 25
}

\begin{abstract}
We apply a newly developed on-the-fly mosaicing technique on the Jansky Very Large Array (VLA) at $3 \mathrm{GHz}$ in order to carry out a sensitive search for an afterglow from the Advanced LIGO binary black hole merger event GW151226. In three epochs between 1.5 and 6 months post-merger, we observed a $100 \mathrm{deg}^{2}$ region, with more than $80 \%$ of the survey region having an rms sensitivity of better than $150 \mu \mathrm{Jy} /$ beam, in the northern hemisphere with a merger containment probability of $10 \%$. The data were processed in near real time and analyzed to search for transients and variables. No transients were found but we have demonstrated the ability to conduct blind searches in a time-frequency phase space where the predicted afterglow signals are strongest. If the gravitational wave event is contained within our survey region, the upper limit on any late-time radio afterglow from the merger event at an assumed mean distance of $440 \mathrm{Mpc}$ is about $10^{29} \mathrm{erg} \mathrm{s}^{-1} \mathrm{~Hz}^{-1}$. Approximately $1.5 \%$ of the radio sources in the field showed variability at a level of $30 \%$, and can be attributed to normal activity from active galactic nuclei. The low rate of false positives in the radio sky suggests that wide-field imaging searches at a few Gigahertz can be an efficient and competitive search strategy. We discuss our search method in the context of the recent afterglow detection from GW170817 and radio follow-up in future gravitational wave observing runs.
\end{abstract}

Key words: gravitational waves - methods: observational - radio continuum: general - surveys

\section{Introduction}

The era of gravitational wave astronomy has begun. The Advanced Laser Interferometer Gravitational-wave Observatory's (aLIGO; Abbott et al. 2016a) first observing run (O1), which ran in the last quarter of 2015, reported two significant binary black hole (BBH) merger events, GW150914 and GW151226 (Abbott et al. 2016b, 2016c). The second observing run of aLIGO (O2) began in late 2016 and ended on 2017 August 25, with the Virgo detector joining on 2017 August 01 to form a three-detector network. Two more significant BBH mergers were detected, GW170104 and GW170814 (Abbott et al. 2017a; The LIGO Scientific Collaboration et al. 2017), and for the first time gravitational waves were detected from the coalesecence of two neutron stars, GW170817 (Abbott et al. 2017b). The notification of the discovery of BBHs triggered a world-wide, panchromatic search for their electromagnetic counterparts (EM; e.g., Abbott et al. 2016d, 2016e; Copperwheat et al. 2016; Cowperthwaite et al. 2016; Evans et al. 2016; Golkhou et al. 2017; Racusin et al. 2017). Thus far, no conclusive time-variable or quiescent emission has been found at any wavelength for BBHs. In contrast, the binary neutron star merger GW170817 was accompanied by EM signals detected at all wavelengths, including prompt gamma-ray emission (Goldstein et al. 2017), fast-fading optical/NIR (Coulter et al. 2017), and delayed X-ray (Troja et al. 2017) and radio emission (Hallinan et al. 2017; Mooley et al. 2018).

Prior to the detection of the EM counterpart to GW170817, radio emission was widely expected to arise on a wide range of

\footnotetext{
${ }^{8}$ Jansky Fellow.
}

timescales and luminosities from compact binary star mergers. Mergers involving neutron stars leave behind significant neutronrich debris that settles into a disk. In the conventional picture, most of the debris disk is accreted by the newly formed black hole post-merger (leading to a short GRB in the case of binary neutron stars) and a small amount, about $0.01 M_{\odot}$, is ejected (e.g., Rosswog et al. 1999, 2013; Bauswein et al. 2013; Hotokezaka et al. 2013; Radice et al. 2016). The forward shock into the ISM swept-up by the (sub-relativistic) ejecta is expected to generate broadband synchrotron emission. This gives rise to a milliJanskylevel radio transient on timescales of months to years (e.g., Nakar \& Piran 2011; Hotokezaka et al. 2016), peaking at frequencies around a few Gigahertz. In cases where relativistic jets are formed and beamed away from the observer, the deceleration of the jet through interaction with the ISM eventually opens up the emission cone into the observer's line of sight. Such orphan afterglows appear as radio transients on timescales of weeks to months (Hotokezaka \& Piran 2015; Hotokezaka et al. 2016). This simple jet model likely needs to be modified as the X-ray and radio emission from GW170817 are best understood as the breakout of wide-angle, mildly relativistic outflow (consistent with a "cocoon") of material entrained by the jet (Gottlieb et al. 2017; Kasliwal et al. 2017; Lazzati et al. 2017; Mooley et al. 2018; Ruan et al. 2017).

For neutron star mergers, there is another possible channel for generating radio emission. A millisecond magnetar is a plausible merger remnant, where the magnetar wind drives a strong shock into the ejecta and the reverse shock results in a "plerion" (cf. the Crab Nebula). A strong plerionic radio emission, which is isotropic 
and independent of the ambient density, is expected on timescales of a few months (Piro \& Kulkarni 2013). There is also a strong radio signal expected at late times ( $\sim$ year timescale; even at small ISM densities) since the magnetar can drive the ejecta to relativistic velocities (e.g., Murase et al. 2017). Past searches for late-time radio emission from short GRBs have put constraints on the phase space of kinetic energy, ejecta mass, and ISM densities, and on the magnetar scenario (Metzger \& Bower 2014; Horesh et al. 2016; Fong et al. 2016).

In the case of binary black holes, radio emission is not widely expected to arise from baryonic poor environments. However, if the $\mathrm{BBH}$ merger launches a relativistic jet into a dense, gas-rich environment, radio emission is expected at a level of the order of 10-100 $\mu \mathrm{Jy}$ (at an assumed distance of $\sim 400 \mathrm{Mpc}$ ), on timescales of $10^{5} \mathrm{~s}$ and at frequencies around a few Gigahertz (Yamazaki et al. 2016; Kashiyama et al. 2017).

There are several challenges that must be overcome in any observational effort designed to detect EM counterparts. At radio wavelengths, the main barriers are achieving the necessary submillijasky sensitivity over the large aLIGO error regions of hundreds of $\mathrm{deg}^{2}$ at Gigahertz frequencies needed to optimally test existing theoretical models. Equally important is having the ability to rapidly identify and reject any variable sources that could lead to misidentification, and to provide subarcsecond localization for any viable candidates (Metzger \& Berger 2012; Metzger et al. 2015; Hotokezaka et al. 2016). In the face of such challenges, several alternative strategies have been adopted. Palliyaguru et al. (2016) and Bhalerao et al. (2017) have taken the approach of carrying out radio follow-up of optically selected candidates, identified from wide-field imaging surveys such as the Dark Energy Camera (DECam; Cowperthwaite et al. 2016) or the Palomar Transient Factory (PTF; Law et al. 2009). This approach avoids imaging large sky areas and it can make deep observations over a wide frequency range. Another approach has been to shift to lower frequencies in order to utilize existing wide-field instruments. Radio searches spanning 50-100 deg ${ }^{2}$ were carried out with the Australian Square Kilometre Array Pathfinder (ASKAP; Bannister et al. 2016), the Low-Frequency Array (LOFAR; Broderick et al. 2016a, 2016b), and the Murchison Widefield Array (MWA; Kaplan et al. 2016) telescopes at frequencies of $863 \mathrm{MHz}, 145 \mathrm{MHz}$, and $118 / 154 \mathrm{MHz}$, respectively. While these surveys did not have the sensitivity and angular resolution needed to detect and provide arcsecond-localization of any putative radio afterglows of the GW events, they were good proof-of-concept experiments for the follow-up of aLIGO sources using wide-field blind radio observations.

In this paper, we take a more direct approach by using the VLA On-the-Fly mosaicing capability to make sensitive, wide-field observations at arcsecond resolution and at Gigahertz frequencies. We use this new mode to carry out follow-up observations of GW151226. In Section 2, we describe the VLA observations and the data reduction carried over a $100 \mathrm{deg}^{2}$ region. The search for transients and the identification of variables is described in Section 3. This search is discussed in the context of future aLIGOVirgo observing runs and radio follow-up programs in Section 4.

\section{Observations and Data Processing}

\subsection{The Gravitational Wave Event GW151226}

The GW151226 gravitational wave signal was initially identified by the GstLAL compact binary coalescence search
(Messick et al. 2017) of the data from the LIGO Hanford and Livingston detectors at 2015 December 26.15, and localized by the BAYESTAR code (Singer \& Price 2016), which is sensitive to compact binary star coalescence events. The false alarm rate for this event was reported as being lower than one per hundred years (Usman et al. 2016; Messick et al. 2017). Using Bayesian Markov-chain Monte Carlo and nested sampling to perform forward modeling of the full GW signal, including spin precession and regression of systematic calibration errors (LALInference; Veitch et al. 2015), the event was localized to within $1240 \mathrm{deg}^{2}$ (90\% credible region), significantly improving over the BAYESTAR localization. The LALInference sky map of the gravitational wave event, together with our VLA survey region, is shown in the left panel of Figure 1.

GW151226 marks the second direct detection of gravitational waves. Detailed offline analysis of the aLIGO data recovered the gravitational wave signal with a significance greater than $5 \sigma$. The initial (individual) and final black hole masses were estimated to lie between 5-22 $M_{\odot}$ and 19-27 $M_{\odot}$, respectively. The luminosity distance was estimated to lie between 250 and $620 \mathrm{Mpc}$, i.e., $0.05<z<0.12$ (the ranges represent the $90 \%$ credible interval; Abbott et al. 2016f).

\subsection{On-the-fly Mosaicing}

The recent refurbishment of the VLA has increased its instantaneous sensitivity by almost an order of magnitude (Perley et al. 2009) but its field of view (several arcmin FWHM) at Gigahertz frequencies is still relatively small. As a result, in the conventional pointed observing mode the slew-and-settle time of the antennas can become a significant fraction of the on-sky integration time, especially for wide-field imaging. These overheads can be minimized through the use of on-the-fly mosaicing (OTFM), where the antennas are driven at a nonsidereal rate and visibilities are recorded continuously, to significantly improve the efficiency of wide-field surveys. OTFM is therefore naturally the observing mode of choice for blind transient searches and LIGO follow-up observations, both of which require observations over wide fields of view and over multiple epochs. One additional advantage of the OTFM method over wide-field radio telescopes is that the VLA can be made to image irregularly shaped GW error regions, with no loss in sensitivity.

The OTFM observing mode (K. P. Mooley et al. 2018, in preparation) has recently been commissioned on the VLA, and this mode can increase the observing efficiency by $\gtrsim 10 \%$ for surveys not requiring high sensitivity (10-100s of $\mu \mathrm{Jy}$ rms noise is sufficient). In the VLA implementation of OTFM, the antennas slew with a uniform speed along a long "stripe" usually in constant R.A. or decl. The antennas are then stepped in decl. or R.A. to the next stripe and so on, in order to observe the survey region in a "basket-weave" pattern of the antenna motion.

\subsection{Radio Observations}

Using the aLIGO LALInference sky localization map for GW151226, we selected a $100 \mathrm{deg}^{2}$ maximum-probability region ${ }^{9}$ in the northern hemisphere (see Figure 1), having a containment

\footnotetext{
9 Bounding coordinates are $\left(\alpha=50^{\circ} .25, \delta=38.25\right),(54.25,34.30),(70.00$ $51.50)$, and $(74.50,47.50)$. The survey area of $100 \mathrm{deg}^{2}$ was motivated by our minimum sensitivity requirement, $\sim 100 \mu \mathrm{Jy}$ per epoch, in order to catch any putative radio afterglow, keeping in consideration also the allotted VLA time for our observations. Furthermore, choosing an additional $50 \mathrm{deg}^{2}$ of highprobability area increased the containment probability only by $0.1 \%$; therefore, we did not increase the survey area beyond $100 \mathrm{deg}^{2}$.
} 

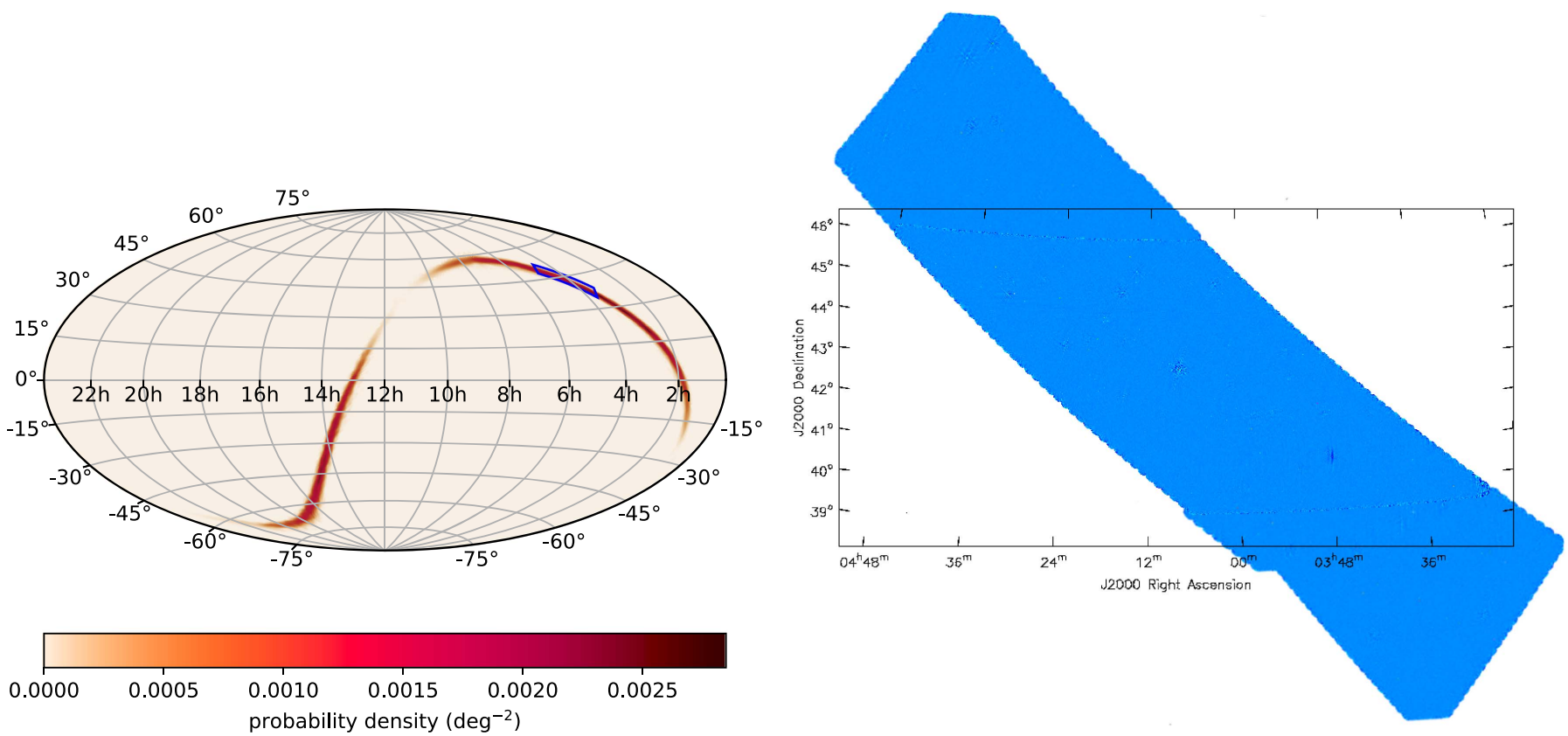

Figure 1. Left: the LALInference localization of GW151226. The $90 \%$ credible region is $1240 \mathrm{deg}^{2}$. The orange colorscale represents the containment probability (black for maximum probability and white for the least). The $100 \mathrm{deg}^{2}$ region observed with the Jansky VLA, having a containment probability of $10 \%$, is outlined in blue. Right: our radio image mosaic of this region from the first observing epoch. The colorbar runs from $100 \mu \mathrm{Jy}$ (blue) to $1500 \mu \mathrm{Jy}$ (white). The median image noise is $\sim 110 \mu \mathrm{Jy} / \mathrm{bm}$ image noise. The noise is higher around bright sources and edges of the survey region.

Table 1

Observing Log

\begin{tabular}{lcccccccc}
\hline \hline No. & $\begin{array}{c}\text { Start Date } \\
(\mathrm{UT})\end{array}$ & Reg/Epo & $\begin{array}{c}\text { LST } \\
(\mathrm{hr})\end{array}$ & $\begin{array}{c}\Delta t \\
(\text { days })\end{array}$ & $\begin{array}{c}\text { Array } \\
\text { Config. }\end{array}$ & $\begin{array}{c}\mathrm{rms} \\
(\mu \mathrm{Jy} / \mathrm{bm})\end{array}$ & $\begin{array}{c}\text { Beam } \\
\left({ }^{\prime \prime}\right)\end{array}$ & Phase Calibrator Sources \\
\hline 1 & 2016 Feb 11 & R2E1 & $00.0-03.8$ & 47 & $\mathrm{C}$ & $\ldots$ & 8 & $\mathrm{~J} 0348+3353, \mathrm{~J} 0414+3418, \mathrm{~J} 0438+4848$ \\
2 & 2016 Feb 14 & R1E1 & $22.5-02.3$ & 50 & $\mathrm{C}$ & 115 & 8 & $\mathrm{~J} 0354+4643, \mathrm{~J} 0438+4848, \mathrm{~J} 0439+4609$ \\
3 & 2016 Apr 05 & R1E2 & $06.0-09.8$ & 101 & $\mathrm{C}$ & $\ldots$ & 8 & $\mathrm{~J} 0354+4643, \mathrm{~J} 0438+4848, \mathrm{~J} 0439+4609$ \\
4 & 2016 Apr 05 & R2E2 & $05.0-08.8$ & 101 & $\mathrm{C}$ & 112 & 8 & $\mathrm{~J} 0348+3353, \mathrm{~J} 0414+3418, \mathrm{~J} 0438+4848$ \\
5 & 2016 Jun 27 & R1E3 & $22.6-02.4$ & 184 & $\mathrm{~B}$ & $\ldots$ & 3 & $\mathrm{~J} 0354+4643, \mathrm{~J} 0438+4848, \mathrm{~J} 0439+4609$ \\
6 & 2016 Jun 30 & R2E3 & $06.1-09.9$ & 187 & B & 150 & 3 & $\mathrm{~J} 0348+3353, \mathrm{~J} 0414+3418, \mathrm{~J} 0438+4848$
\end{tabular}

Notes. (1) Entries from left to right include the observing run (No.), the start date, the survey region and epoch (Reg/Epo), the start and stop LST time, the time in days since the GW event, the VLA array configuration, the rms noise for each epoch, the synthesized beam size and a list of phase calibrators used for each epoch. (2) rms refers to the 50th percentile of the rms noise across the survey region for the given epoch.

probability of $10 \%$, for follow-up. Observations were carried out ${ }^{10}$ across three epochs (E1, E2, E3) with the Karl G. Jansky Very Large Array (VLA) in the B and C array configurations. S-band (2-4 GHz) was chosen to maximize survey speed and catch a putative late-time afterglow. To maximize the continuum imaging sensitivity, we used the Wideband Interferometric Digital Architecture (WIDAR) correlator with 16 spectral windows, 64 $2 \mathrm{MHz}$ wide channels each to get $2 \mathrm{GHz}$ of total bandwidth centered on $3.0 \mathrm{GHz}$. Two basebands, centered on $2.5 \mathrm{GHz}$ and $3.5 \mathrm{GHz}$, consisted of eight spectral windows each. We used the OTFM mode (Section 2.2) and used $1 \mathrm{~s}$ integrations to minimize the amplitude smearing. A $\log$ of the observations is given in Table 1.

In order to facilitate the scheduling, we divided the survey area into two regions, R1 (decl. 39.5-46.0) and R2 (decl. $34^{\circ} .2-39^{\circ} .5$ and $46^{\circ} .0-51^{\circ} .5$ ), which were observed in each epoch within a span of a few days. Our dynamically scheduled observing blocks (for each region and each epoch) were $\sim 3.75$ $\mathrm{hr}$ and were designed using OTFSim (K. P. Mooley et al. 2018,

${ }^{10}$ Under project code VLA/16A-237. in preparation). Given these boundaries of the survey region, OTFSim automatically selected the appropriate path of antennas and complex gain calibrators to minimize the slew time. The antenna slewing was done along constant decl. in order to ensure uniform coverage and sensitivity over the survey region. Thus, we designed our OTFM observations to slew the antennas purely in R.A. at a rate of 2 arcmin per second (on-sky rate of 1.6 arcmin per second), stepping northwards by 10.6 arcmin (FWHM of primary beam divided by $\sqrt{2}$, to get approximately uniform rms noise across the survey region) after each slew, in a basket-weave fashion. The correlator phase center was stepped every 4 arcmin in R.A. (every $2 \mathrm{~s}$ ) to ensure that the antenna slew during each scan in the observation was well within one full primary beam. 3C147 was used as the flux density and bandpass calibrator.

\subsection{RFI Flagging, Calibration, Imaging, and Source Finding}

Immediately after the observations for each epoch/region (each observing block) were complete, we downloaded the raw data from the VLA archive, onto the lustre file system at the 
NRAO AOC in Socorro. The data were then calibrated using the NRAO scripted pipeline (in CASA 4.5.0) with quacking ${ }^{11}$ of the target fields removed. Due to substantial satellite-induced RFI, the data between 2.12 and $2.37 \mathrm{GHz}$ (the second and third spectral windows) were fully flagged for all observations. Single scans (5500-5700 scans for each region per epoch) were then split from the calibrated data set into individual measurement sets. The calibration and splitting processes for each observing block took about $36 \mathrm{hr}$.

We then ran a distributed imaging process on 70 cores across five computer nodes at the NRAO AOC using CASA task clean, such that each core was imaging a single scan at any given time. To enable linear mosaicing, we convolved the images with fixed circular synthesized beams ${ }^{12}$ of 8 arcsec (for the $\mathrm{C}$ config observations) or 3 arcsec (for the $\mathrm{B}$ config observations). The imaging of each scan was itself done over two iterations. In the first iteration, we cleaned using Briggs weighting and a robust parameter of zero in order to minimize imaging artifacts. Images with sources brighter than $20 \mathrm{mJy}$ were then used to derive and apply a single phase selfcalibration procedure. Clean boxes were then derived based on the sources found using the ia.findsources tool in CASA. This was followed by clipping of the visibilities in amplitude (in all spectral windows; in order to minimize the effect of RFI) at a level equal to the sum of the mean and three times the standard deviation of the visibility amplitudes in the spectral window with the least RFI. The second imaging iteration used the self-calibrated visibilities to image using natural weighting and clean boxes. During each cleaning step, we used 700 clean iterations, two Taylor terms, a cyclefactor parameter of 7, and a clean stopping threshold of $0.3 \mathrm{mJy}$. The pixel size was chosen so as to sample the synthesized beam across at least four pixels. The images had a center frequency of $3.0 \mathrm{GHz}$, except for the observation on 2017 June 27 (where the data had substantial RFI between 2 and $3 \mathrm{GHz}$ ) for which the center frequency is $3.3 \mathrm{GHz}{ }^{13}$ A small fraction of the single-scan images had strong spike-shaped artifacts (there is a known bug in CASA clean when using two or more Taylor terms, which is currently being fixed), and for these scans we chose the Briggsweighted images (output during the first imaging iteration described above) instead of the ones with two Taylor terms. Based on our inspection of the single-scan images, we expect that the Briggs weighting reduces the integrated flux density of extended sources in the survey region by a fraction less than or equal to $\sim 10 \%$. For unresolved sources, there will be no change in flux density due to the introduction of the Briggsweighted images.

Linear mosaicing of the single-scan images was then carried out using FLATN in AIPS, followed by cropping of the mosaic into $4096 \times 4096$ pix $^{2}$ subimages. We used the primary beam parameters from Perley et al. (EVLA Memo 195) during the linear mosaicing step. The imaging and linear mosaicing processes together required about $6 \mathrm{hr}$ per region/epoch. We then made a background noise map for each subimage using RMSD in AIPS, which was then supplied to SAD for the

\footnotetext{
11 Quacking is the process of removing (potentially bad) data from the beginning and end of each scan.

${ }^{12}$ The synthesized beam sizes found by clean ranged between $\sim 7^{\prime \prime}$ and $9^{\prime \prime}$ for $\mathrm{C}$ config data and between $\sim 2^{\prime \prime}$ and $3^{\prime \prime}$ for B config data.

13 For sources with steep spectral indices of \pm 1 , the flux densities between the $3 \mathrm{GHz}$ images and the $3.3 \mathrm{GHz}$ images will differ by $\sim 10 \%$. This biases our variability search slightly in favor of finding steep spectral index sources, as discussed in more detail in Section 3.
}

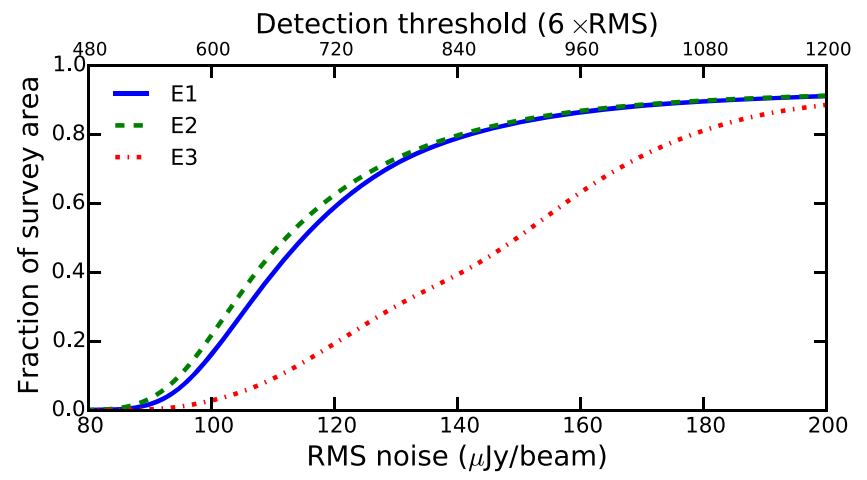

Figure 2. Cumulative plots of the rms noise across the survey region for the three epochs of observations reported here. The source detection threshold $(6 \sigma)$ is shown on the upper $x$-axis. About $10 \%$ of the survey region, corresponding to areas around bright sources and field edges, have rms noise $>200 \mu \mathrm{Jy} / \mathrm{beam}$.

cataloging of sources down to ${ }^{14} 6 \sigma$. The cumulative noise plots made from the background rms noise maps is shown in Figure 2.

As we are interested in only unresolved and partially resolved sources, we chose only those sources from each epoch that had an integrated-to-peak flux density ratio of $<1.5$ and prepared a merged catalog, consisting of 5307 sources. For sources in the merged catalog that were not detected in some of the epochs, we set the peak flux density to be equal to the peak pixel value at the location of the source. For those sources that have integrated flux density lower than the peak flux density, we set the integrated flux density to be equal to the latter. This cataloging process required about $1 \mathrm{hr}$. The total time required from downloading the raw data for each observing block and producing a final source catalog was $43 \mathrm{hr}$.

\section{Transient and Variability Search}

We used the catalog of 5307 sources from Section 2.4 to carry out a search for transient sources that appeared or disappeared in one or more of the three epochs. No transients were found to a $6 \sigma$ limit of $670 \mu \mathrm{Jy}$ (50\% completeness threshold for the merged catalog over three epochs and $100 \operatorname{deg}^{2}$ ).

The same catalog was used to investigate variability following a process described in more detail in Mooley et al. (2016). In short, for each source, we calculate the variability statistic, $V_{s}=\Delta S / \sigma$ for each epoch and a modulation index $m=\Delta S / \bar{S}$, where $S$ is the flux density, $\bar{S}$ is the average flux density, $\Delta S$ is the flux density difference, and $\sigma$ is the rms noise. Significant variables were identified as those sources that have $V_{s}$ larger than $4 \sigma$, and the absolute value of the modulation index, $|m|$, larger than 0.26 (i.e., a fractional variability, $\left.f_{\text {var }}>0.3\right)$. The constraint on $V_{s}$ ensures fewer than one false positive will be detected as a variable source in our search, assuming Gaussian statistics. Considering that our flux scale is accurate to only 3\%-5\% (Thyagarajan et al. 2011; Mooley et al. 2013), the artificial variability induced on account of our imaging parameters ( $\sim 10 \%$; see Section 2.4 and also Mooley et al. 2016), and our usage of the true primary beam instead of the smeared OTFM beam, the constraint on the

\footnotetext{
${ }^{14}$ For the B config data, there are $2 \times 10^{8}$ synthesized beams across the 100 $\operatorname{deg}^{2}$ survey region. So the $6 \sigma$ detection threshold ensures $<1$ false detection due to noise (assuming Gaussian statistics).
} 
fractional variability is needed to minimize the number of false positives.

We used epoch E1 as the reference epoch and compared E2 and E3 independently with this epoch using the $V_{s}$ and $m$ for finding significant variable sources. Based on our inspection of the ratios of source flux densities between E2 and E1, we had to apply a multiplicative correction factor of 1.07 to the source flux densities in E2. Similarly, our comparison of E3 versus E1 instructed us to apply a multiplicative correction factor ${ }^{15}$ of 1.30 to the source flux densities in E3. This correction factor made the distribution of the variability statistic $V_{s}$ symmetrical, but also broadened it to a Gaussian-like distribution $(\sigma=2.5$, hence our $4 \sigma$ variability selection criterion in this case is $V_{s}>10$ ) rather than a Student-t distribution (see Figure 9 of Mooley et al. 2016). During the variability search between E3 and E1, we also discovered that a significant fraction of the sources in the final source catalog were resolved out in E3, and an abnormally large number of variables appeared below $\sim 1$ mJy (this can again be attributed largely to the angular resolution differences). Hence, for the comparison between E3 and E1 we restricted our search to only those sources (total of 2782) having an integrated-to-peak flux density ratio ${ }^{16}$ of $<1.2$.

The plot of the variability statistic versus the modulation index is shown in Figure 3. We found 72 significant variable sources between E1 and E2 and 42 variables between E1 and E3, having fractional variability larger than $30 \%$. This indicates a variability fraction of $1.5 \pm 0.2 \%$ for timescales between a few weeks and a few months.

\section{Discussion and Future Prospects}

We have used the VLA to image a $100 \mathrm{deg}^{2}$ error region of the O1 aLIGO BBH merger event GW151226. While a bright EM counterpart was not expected in this case, we have demonstrated a near real time ability to conduct blind searches in a phase space where the predicted afterglow signature is strongest. As we noted in Section 1, a late-time radio afterglow peaking on timescales of hundreds of days is one of the more robustly predicted afterglow signatures from neutron star mergers (Nakar \& Piran 2011). Such searches are best carried out at Gigahertz frequencies, since synchrotron self-absorption suppresses the signal below $1 \mathrm{GHz}$ for up to several years postmerger. Estimates of the radio afterglow signal currently suffer from uncertainties in the circum-merger gas density, and a full search must be sensitive to the low densities $\left(10^{-3} \mathrm{~cm}^{-3}\right)$, as seen for some short gamma-ray bursts (Fong et al. 2014), and the density within galactic disks, $\sim 1 \mathrm{~cm}^{-3}$ (Draine 2011), as expected for the Galactic binary pulsar population.

Metzger \& Berger (2012) have listed the "cardinal virtues" that must be met for an experiment to be considered a competitive follow-up effort for detecting the predicted signatures of compact binary star mergers. These are (1) that they are detectable with current observing facilities with a reasonable time allocation, (2) that they accompany a

\footnotetext{
$\overline{15}$ This factor is rather high, but is partly due to angular resolution difference between the two epochs. The flux lost in E3 is due to the missing short spacings in the VLA B array configuration, and could not be recovered to the $\mathrm{C}$ array flux even after imaging with a restricted UV range and UV tapering. In principle, the missing short spacings should not affect true point sources, but the robust comparison of flux densities across different array configurations is an issue that needs to be investigated further.

16 This constraint somewhat reduces our ability to reliably find variable sources below $\sim 1 \mathrm{mJy}$, but at the same time significantly mitigates false positives.
}

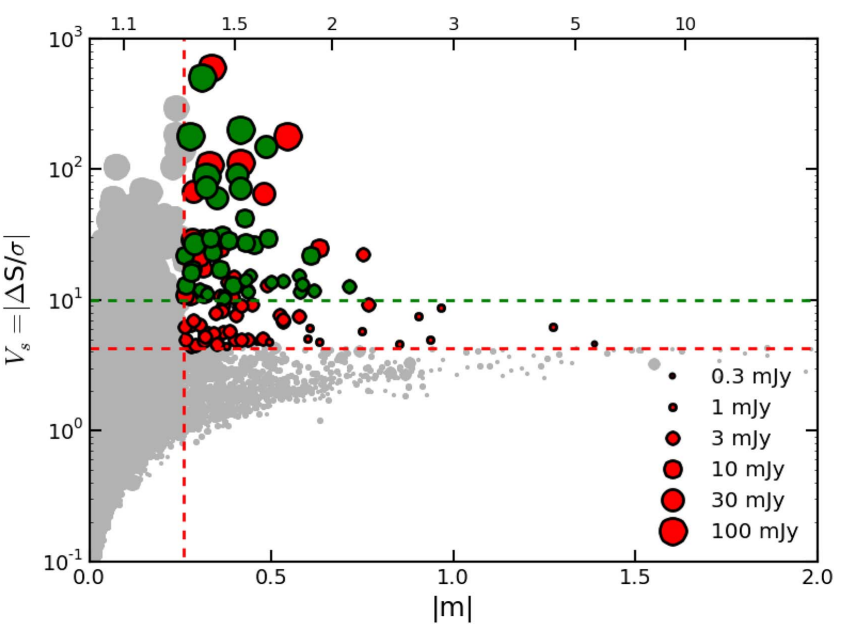

Figure 3. Variability statistic $\left(V_{s}\right)$ vs. the modulation index $(m)$ for the unresolved/partially resolved sources in our merged catalog. Gray points indicate sources (from the E1-E2 and E1-E3 comparisons) that are not significant variables. The red points are the selected variables between E1 and E2 (totaling 72 out of 5307 sources), and green points are those between E1 and E3 (totaling 42 out of 2782 sources). The horizontal red and green dashed lines indicate the variability selection criteria in $V_{s}$ for these two respective cases. The vertical red dashed line is the variability selection criteria in the modulation index. The flux densities of the sources defines the marker size. The top horizontal scale is the fractional variability $f_{\text {var }}$, defined as the ratio of the flux densities of a source between two epochs. See Section 3 for details.

significant fraction of GW events, (3) that they can be distinguished from other astrophysical transients, and (4) that they can provide arcsecond sky localization. Below we demonstrate how this experiment addresses the challenges and meets almost all of these requirements.

Since the field of view of the VLA at $3 \mathrm{GHz}$ is small $\left(0.06 \mathrm{deg}^{2}\right)$, we take advantage of the superb instantaneous sensitivity of the VLA and use the OTFM method, rapidly slewing the antennas $(1.6 /$ minute $)$ in order to image a $100 \mathrm{deg}^{2}$ error region with only $7.5 \mathrm{hr}$ of on-sky integration time. These observations were made at three epochs approximately 50, 101, and 185 days post-merger (see Table 1). These are an appropriate range of timescales for searching for the rise and fall of the expected late-time radio afterglow. The typical rms noise achieved for each epoch was approximately $120 \mu \mathrm{Jy} /$ beam. This is an astrophysically interesting sensitivity level for detecting afterglows circum-merger gas densities $>0.1 \mathrm{~cm}^{-3}$ and distances of 100-200 Mpc (Hotokezaka et al. 2016).

We searched for any transient sources that appeared in the $100 \mathrm{deg}^{2}$ region over the three epochs. None were found. This implies an upper limit to the areal density of transients brighter than $700 \mu \mathrm{Jy}$ of $10^{-2} \mathrm{deg}^{-2}$. Our limit is similar to that obtained from the $3 \mathrm{GHz}$ Stripe 82 Pilot project (Mooley et al. 2016). The types and timescales of the different radio transients at frequencies of a few Gigahertz are shown in Figure 4. On the timescales that were sampled in this experiment, the expected transients include tidal disruption events (TDE), orphan GRB afterglows, and Type II corecollapse supernovae. If the gravitational wave event is contained within our survey region, we can place an upper limit of about $10^{29} \mathrm{erg} \mathrm{s}^{-1} \mathrm{~Hz}^{-1}$ to any late-time radio afterglow from the merger event at an assumed mean distance of $440 \mathrm{Mpc}$. At an areal density of $10^{-2} \mathrm{deg}^{-2}$, none of these transient source classes are expected to be a significant contaminant in a blind radio survey for the late-time afterglow from a binary compact remnant merger Mooley et al. (2016). 


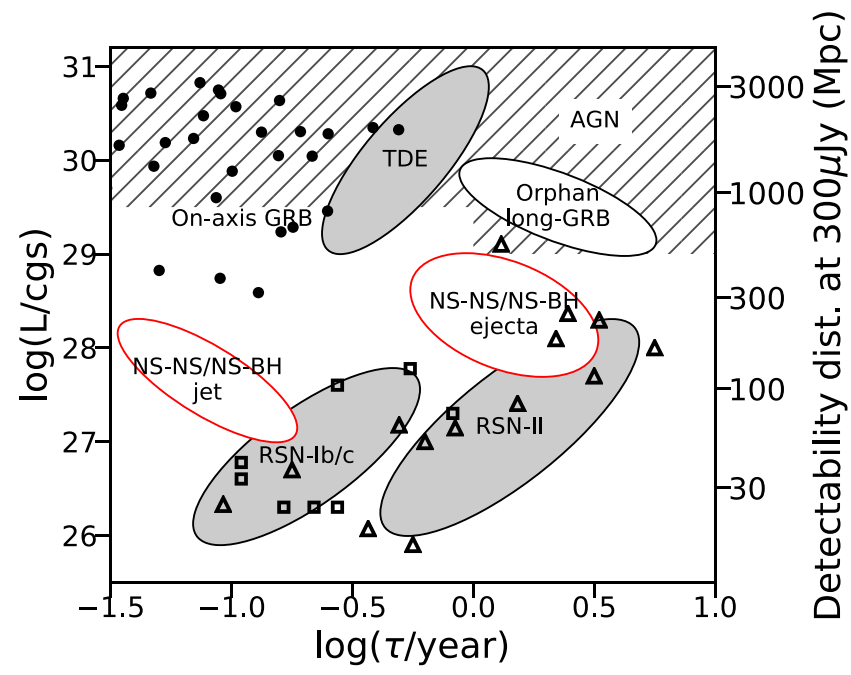

Figure 4. Luminosity (in erg $\mathrm{s}^{-1} \mathrm{~Hz}^{-1}$ ) vs. timescale phase space of radio transients at frequencies of a few Gigahertz. The late-time afterglows of NSNS/NS-BH mergers (red ellipses) are some of the best electromagnetic counterparts expected for aLIGO sources (Hotokezaka et al. 2016). They are distinguished from other transient classes (black ellipses; shaded region represents AGN) in this phase space. Some overlap exists with Type II supernovae, but the latter are expected to be optically thick at peak, while the former will be optically thin. Filled circles, open triangles, and squares represent on-axis GRBs (Chandra \& Frail 2012), Type II and Type Ib/c SNe (Weiler et al. 2002; Stockdale et al. 2006; Soderberg et al. 2010), respectively, followed up in the radio. Additionally, the low transient rates imply low contamination by false positives. Note that the (early-time) radio afterglow of GW170817 has a luminosity of $\sim 10^{26}$ and a timescale of $>1$ month (Hallinan et al. 2017; Mooley et al. 2018). The right y-axis denotes the detectability distance for a deeper survey than ours, having a detection threshold of $300 \mu \mathrm{Jy}$. Figure adapted from Frail et al. (2012) and Mooley et al. (2016).

The quietness of the radio sky stands in contrast to optical counterpart searches (e.g., Copperwheat et al. 2016; Cowperthwaite et al. 2016; Palliyaguru et al. 2016), for which there were a number of transients that required spectroscopic follow-up before being eliminated as candidates. Radio observations of GW sources also have an advantage over the optical and X-ray in cases where solar/lunar observing constraints are present, and dust-obscured environments in the host galaxy itself, or along lines of sight through our own Galaxy. For example, continued radio monitoring of GW170817 was carried out during a crucial period in the evolution of the afterglow light curve, while optical and X-ray telescopes were constrained by the Sun, thus allowing us to distinguish between the off-axis jet versus cocoon models (Mooley et al. 2018).

While no transients were found, about $1.5 \%$ of the compact, persistent radio sources in the field exhibited significant variability. Most radio sources vary to some degree or another. Below $\sim 1 \mathrm{GHz}$, the variations are dominated by plasma effects such as interstellar scintillation, while at high frequencies the variations are intrinsic, likely originating from changes in the black hole-disk-jet environments (Altschuler 1989). The frequency and level of variability is in agreement with previous Gigahertz studies (Mooley et al. 2013, 2016; Hancock et al. 2016) and is consistent with normal AGN activity ${ }^{17}$ on a timescale of a few months. In principle, a transient occurring in a star-forming galaxy could be mistakenly identified as an

\footnotetext{
17 We have manually inspected the optical/infrared images and photometric colors of the variable sources having multiwavelength counterparts and crossmatched with AGN catalogs to confirm that these are indeed AGNs.
}

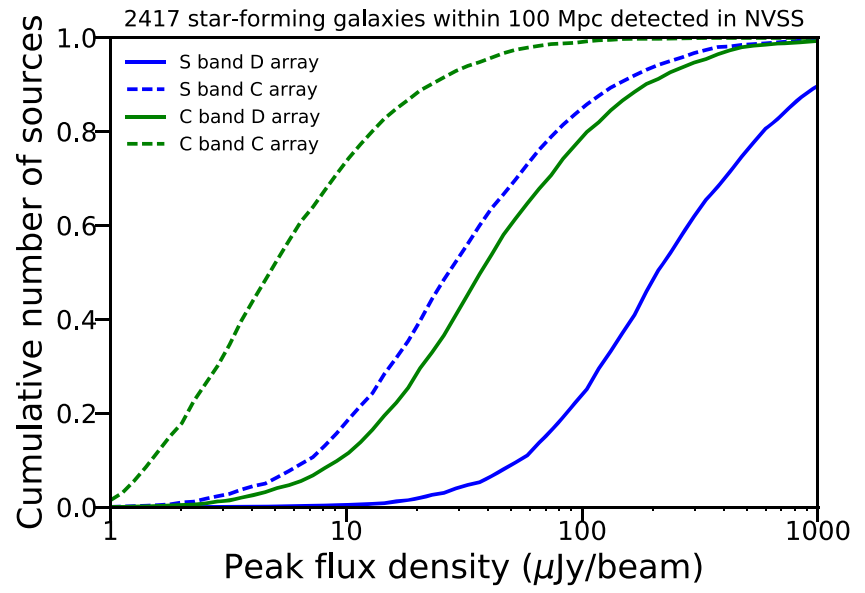

Figure 5. Cumulative plots for the peak flux densities of star-forming galaxies within $100 \mathrm{Mpc}$ (detected in the NVSS; Condon et al. 2002) at S and C bands $(3 \mathrm{GHz}$ and $6 \mathrm{GHz}$ respectively) for the VLA C and D array configurations. Contamination by the host galaxy may be a significant problem for merger afterglows in $\mathrm{D}$ config, but searches at $\mathrm{C}$ band will abate this. Beyond 100-150 Mpc, contamination becomes less severe (Hotokezaka et al. 2016). If the merger afterglows reside within the galactic disks, they will make the host galaxies appear as variable sources. Two fiducial surveys with source detection thresholds of $100 \mu \mathrm{Jy}$ and $300 \mu \mathrm{Jy}$ will be able to reliably recover variable point sources in host galaxies having peak flux densities less than $\sim 400 \mu \mathrm{Jy}$ and $\sim 1200 \mu \mathrm{Jy}$ (i.e., corresponding to $25 \%$ variability). This corresponds to $70 \%(95 \%)$ and $90 \%(100 \%)$ of the host galaxies, respectively, within $100 \mathrm{Mpc}$ at $\mathrm{S}$ band (C band) in $\mathrm{D}$ config. An alternative, but more challenging, method will be to employ host-galaxy subtraction. In this plot, correction for the observing frequency has been made assuming a spectral index of -0.7 . Integrated flux density and size of the radio source have been converted to the peak flux density using the equation for face-on galaxy from Condon (2015).

unresolved or partially resolved variable source. In practice, with the 8 arcsecond synthesized beam and the rms noise of $\sim 120 \mu \mathrm{Jy} /$ beam, the experiment does not have sufficient surface brightness sensitivity to detect star-forming galaxies within the LIGO volume (Condon 2015) for GW151226. However, host-galaxy contamination may be severe in the cases where merger afterglows are located within $\sim 100 \mathrm{Mpc}$ and observations are carried out in VLA D config (see Figure 5). Hotokezaka et al. (2016) looked at contamination as a function of galaxy distance for VLA B config and L band $(1.4 \mathrm{GHz})$, and they find that contamination due to starburst emission and bright AGNs will be $\sim 5 \%$. Nevertheless, an interferometer such as the VLA not only provides a subarcsecond position for a potential merger event, but it also acts as a spatial filter of extended host-galaxy emission.

While we have shown that Gigahertz radio searches for late afterglows are a promising method to search for the EM signature of a GW event, the experiment could still be improved. In Section 2.4, we noted that 1.8 days were required to process each epoch. Lower latency would be possible if the data calibration stage was further optimized. The benefits of such improvements are not immediately obvious. The radio afterglow reaches its peak months after the gravitational event, well after any putative optical signature has faded away. The follow-up of any radio candidate would be with a goal of identifying a host galaxy and measuring its redshift, and as such could be done at a more leisurely pace.

A weakness of radio searches is that the large uncertainty in the expected circum-merger densities predicts a wide range of peak flux densities at Gigahertz frequencies (see Section 1). Figure 6 shows the cumulative distributions of peak flux 


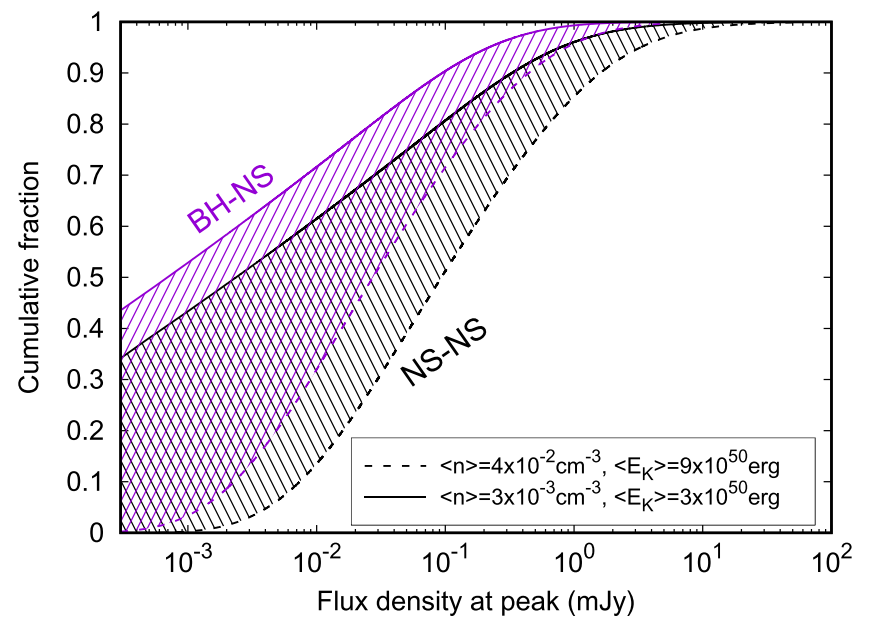

Figure 6. Cumulative flux density distributions expected for double neutron star (NS-NS; black region) and black hole neutron star (BH-NS; purple region) mergers (those detected as gravitational wave sources) at the peaks of their light curves. The distributions suggest that blind radio searches with rms noise values of $50-75 \mu \mathrm{Jy} /$ beam will be able to access up to $40 \%$ of the merger afterglows. For calculating these curves, we use the density distribution derived from short-GRB afterglow observations (similar to the $\nu_{c}<\nu_{x}$ case for an optimistic choice and the $n_{\min }=10^{-6} \mathrm{~cm}^{-3}$ case for a pessimistic choice; Fong et al. 2015). We use a hypothetical log-normal distribution for the kinetic energy with a peak at $9 \times 10^{50}\left(3 \times 10^{50}\right)$ erg for an optimistic (pessimistic) choice and the standard deviation chosen to be unity. The distribution of the distances of binary neutron and black hole neutron star mergers is taken from Hotokezaka et al. (2016). The microphysical parameters are set to: $\epsilon_{e}=\epsilon_{B}=0.1$ and $p=2.5$. Note that the only difference between the NS-NS and BH-NS cases (as plotted) is the distance distribution; the latter occur at farther distances so their flux densities are lower. However, the kinetic energies involved in BH-NS mergers may be larger, depending on the black hole spins, mass ratios, and neutron star equation of state.

densities of neutron star merger (simulated population of gravitational wave sources; Hotokezaka et al. 2016). It is possible to achieve noise values of 50-75 $\mu \mathrm{Jy} /$ beam and thus broaden the parameter space of the late-time afterglow models. Such deeper searches will be able to access up to $40 \%$ of the merger afterglows. For this blind survey approach, and with finite VLA telescope time, the trade off is to image smaller fields of view. Such decreases in the size of the GW error regions are expected in the upcoming runs jointly with LIGO and Virgo, and eventually runs including KAGRA and LIGO-India (Nissanke et al. 2013; Kasliwal \& Nissanke 2014; Abbott et al. 2016g). Another approach for increasing sensitivity is to significantly reduce the total area searched by doing targeted searches. This approach involves deep radio follow-up of transient candidates identified at other wavelengths (Palliyaguru et al. 2016; Bhalerao et al. 2017) or using a master galaxy catalog in the localized volume (Gehrels et al. 2016; Singer et al. 2016). Such a strategy has been successful in the case of GW170817. However, as the distance range for the gravitational wave detectors increases, blind surveys or even hybrid approaches between targeted and blind searches will become important.

The National Radio Astronomy Observatory is a facility of the National Science Foundation operated under cooperative agreement by Associated Universities, Inc. K.P.M. acknowledges support from the Oxford Centre for Astrophysical Surveys, which is funded through the Hintze Family Charitable Foundation. K.P.M. is currently a Jansky Fellow of the National Radio Astronomy
Observatory. We thank the anonymous referee for valuable comments that helped improve this manuscript.

\section{ORCID iDs}

K. P. Mooley (iD https://orcid.org/0000-0002-2557-5180

S. R. Kulkarni (iD https://orcid.org/0000-0001-5390-8563

K. Hotokezaka (ib https://orcid.org/0000-0002-2502-3730

L. P. Singer (1) https://orcid.org/0000-0001-9898-5597

M. M. Kasliwal (iD https://orcid.org/0000-0002-5619-4938

S. B. Cenko (D) https://orcid.org/0000-0003-1673-970X

\section{References}

Abbott, B. P., Abbott, R., Abbott, T. D., et al. 2016a, PhRvL, 116, 131103 Abbott, B. P., Abbott, R., Abbott, T. D., et al. 2016b, PhRvL, 116, 061102 Abbott, B. P., Abbott, R., Abbott, T. D., et al. 2016c, PhRvL, 116, 241103 Abbott, B. P., Abbott, R., Abbott, T. D., et al. 2016d, ApJL, 826, L13 Abbott, B. P., Abbott, R., Abbott, T. D., et al. 2016e, ApJS, 225, 8 Abbott, B. P., Abbott, R., Abbott, T. D., et al. 2016f, PhRvL, 116, 241103 Abbott, B. P., Abbott, R., Abbott, T. D., et al. 2016g, LRR, 19, 1

Abbott, B. P., Abbott, R., Abbott, T. D., et al. 2017a, PhRvL, 119, 141101 Abbott, B. P., Abbott, R., Abbott, T. D., et al. 2017b, PhRvL, 119, 161101 Altschuler, D. R. 1989, FCPh, 14, 37

Bannister, K., Marvil, J., Heywood, I., et al. 2016, LV-EM GRB Coordinates Network, 18363

Bauswein, A., Goriely, S., \& Janka, H.-T. 2013, ApJ, 773, 78

Bhalerao, V., Kasliwal, M. M., Bhattacharya, D., et al. 2017, arXiv:1706.00024

Broderick, J., Jonker, P., Fender, R., et al. 2016a, LV-EM GRB Coordinates Network, 18364

Broderick, J., Rowlinson, A., Jonker, P., et al. 2016b, LV-EM GRB Coordinates Network, 18809

Chandra, P., \& Frail, D. A. 2012, ApJ, 746, 156

Condon, J. 2015, arXiv:1502.05616

Condon, J. J., Cotton, W. D., \& Broderick, J. J. 2002, AJ, 124, 675

Copperwheat, C. M., Steele, I. A., Piascik, A. S., et al. 2016, MNRAS, 462,3528

Coulter, D. A., Foley, R. J., Kilpatrick, C. D., et al. 2017, arXiv:1710.05452

Cowperthwaite, P. S., Berger, E., Soares-Santos, M., et al. 2016, ApJL, 826, L29

Draine, B. T. 2011, Physics of the Interstellar and Intergalactic Medium (Princeton, NJ: Princeton Univ. Press)

Evans, P. A., Kennea, J. A., Palmer, D. M., et al. 2016, MNRAS, 462, 1591

Fong, W., Berger, E., Margutti, R., \& Zauderer, B. A. 2015, ApJ, 815, 102

Fong, W., Berger, E., Metzger, B. D., et al. 2014, ApJ, 780, 118

Fong, W., Metzger, B. D., Berger, E., \& Özel, F. 2016, ApJ, 831, 141

Frail, D. A., Kulkarni, S. R., Ofek, E. O., Bower, G. C., \& Nakar, E. 2012, ApJ, 747,70

Gehrels, N., Cannizzo, J. K., Kanner, J., et al. 2016, ApJ, 820, 136

Goldstein, A., Veres, P., Burns, E., et al. 2017, ApJL, 848, L14

Golkhou, V. Z., Butler, N. R., Strausbaugh, R., et al. 2017, arXiv:1706.03898

Gottlieb, O., Nakar, E., Piran, T., \& Hotokezaka, K. 2017, arXiv:1710.05896

Hallinan, G., Corsi, A., Mooley, K. P., et al. 2017, Sci, 358, 1579

Hancock, P. J., Drury, J. A., Bell, M. E., Murphy, T., \& Gaensler, B. M. 2016 , MNRAS, 461, 3314

Horesh, A., Hotokezaka, K., Piran, T., Nakar, E., \& Hancock, P. 2016, ApJL, 819, L22

Hotokezaka, K., Kiuchi, K., Kyutoku, K., et al. 2013, PhRvD, 87, 024001

Hotokezaka, K., Nissanke, S., Hallinan, G., et al. 2016, arXiv:1605.09395

Hotokezaka, K., \& Piran, T. 2015, MNRAS, 450, 1430

Kaplan, D. L., Murphy, T., Rowlinson, A., et al. 2016, PASA, 33, e050

Kashiyama, K., Hotokezaka, K., \& Murase, K. 2017, arXiv:1710.10765

Kasliwal, M. M., Nakar, E., Singer, L. P., et al. 2017, arXiv:1710.05436

Kasliwal, M. M., \& Nissanke, S. 2014, ApJL, 789, L5

Law, N. M., Kulkarni, S. R., Dekany, R. G., et al. 2009, PASP, 121, 1395

Lazzati, D., Deich, A., Morsony, B. J., \& Workman, J. C. 2017, MNRAS, 471, 1652

Messick, C., Blackburn, K., Brady, P., et al. 2017, PhRvD, 95, 042001

Metzger, B. D., \& Berger, E. 2012, ApJ, 746, 48

Metzger, B. D., \& Bower, G. C. 2014, MNRAS, 437, 1821

Metzger, B. D., Williams, P. K. G., \& Berger, E. 2015, ApJ, 806, 224

Mooley, K. P., Frail, D. A., Ofek, E. O., et al. 2013, ApJ, 768, 165

Mooley, K. P., Hallinan, G., Bourke, S., et al. 2016, ApJ, 818, 105 
Mooley, K. P., Nakar, E., Hotokezaka, K., et al. 2018, Natur, 554, 207

Murase, K., Toomey, M. W., Fang, K., et al. 2017, arXiv:1710.10757

Nakar, E., \& Piran, T. 2011, Natur, 478, 82

Nissanke, S., Kasliwal, M., \& Georgieva, A. 2013, ApJ, 767, 124

Palliyaguru, N. T., Corsi, A., Kasliwal, M. M., et al. 2016, ApJL, 829, L28

Perley, R., Napier, P., Jackson, J., et al. 2009, IEEEP, 97, 1448

Piro, A. L., \& Kulkarni, S. R. 2013, ApJL, 762, L17

Racusin, J. L., Burns, E., Goldstein, A., et al. 2017, ApJ, 835, 82

Radice, D., Galeazzi, F., Lippuner, J., et al. 2016, MNRAS, 460, 3255

Rosswog, S., Liebendörfer, M., Thielemann, F.-K., et al. 1999, A\&A, 341, 499

Rosswog, S., Piran, T., \& Nakar, E. 2013, MNRAS, 430, 2585

Ruan, J. J., Nynka, M., Haggard, D., Kalogera, V., \& Evans, P. 2017, arXiv: 1712.02809

Singer, L. P., Chen, H.-Y., Holz, D. E., et al. 2016, ApJL, 829, L15
Singer, L. P., \& Price, L. R. 2016, PhRvD, 93, 024013

Soderberg, A. M., Chakraborti, S., Pignata, G., et al. 2010, Natur, 463, 513

Stockdale, C. J., Maddox, L. A., Cowan, J. J., et al. 2006, AJ, 131, 889

The LIGO Scientific Collaborationthe Virgo Collaboration, Abbott, B. P., et al. 2017, arXiv:1706.01812

Thyagarajan, N., Helfand, D. J., White, R. L., \& Becker, R. H. 2011, ApJ, 742, 49

Troja, E., Piro, L., van Eerten, H., et al. 2017, Natur, 551, 71

Usman, S. A., Nitz, A. H., Harry, I. W., et al. 2016, CQGra, 33, 215004

Veitch, J., Raymond, V., Farr, B., et al. 2015, PhRvD, 91, 042003

Weiler, K. W., Panagia, N., Montes, M. J., \& Sramek, R. A. 2002, ARA\&A, 40, 387

Yamazaki, R., Asano, K., \& Ohira, Y. 2016, PTEP, 2016, $051 E 01$ 